\title{
Numerical analysis of broad band fiber optical parametric amplifiers pumped by two chirped pulses
}

\author{
Damien Bigourd, ${ }^{1, *}$ Coralie Fourcade Dutin, Olivier Vanvincq, ${ }^{2}$ and Emmanuel Hugonnot ${ }^{3}$ \\ ${ }^{1}$ Institut FEMTO-ST, Département d'Optique, UMR 6174, \\ Université Bourgogne Franche-Comté-CNRS, 25030 Besançon, France \\ ${ }^{2}$ Laboratoire PhLAM, Physique des Lasers Atomes et Molécules, \\ UMR 8523, Université Lille-CNRS, 59655 Villeneuve dAsq, France \\ ${ }^{3}$ Commissariat à l'Énergie Atomique et aux Énergies Alternatives, \\ Centre d'Études Scientifiques et Techniques d'Aquitaine, 33116 Le Barp Cedex, France
}

compiled: June 29, 2016

\begin{abstract}
Optical parametric amplifier pumped by two chirped pump pulses has been analytically and numerically investigated to predict the spectral gain bandwidth and its temporal distribution. We highlight that the properties of the amplifier depend strongly on the relative chirp values and a uniform spectro-temporal gain can be obtained when the two chirps have opposite signs. A chirped signal pulse owing a very broad bandwidth has also been injected and we demonstrate the amplification of ultra-short pulse with this type of amplifiers.

OCIS codes: (190.4410) Nonlinear optics, parametric processes; (190.4380) Nonlinear optics, fourwave mixing; (060.4370) Nonlinear optics, fibers.(140.3490) Lasers, distributed-feedback

http://dx.doi.org/10.1364/XX.99.099999
\end{abstract}

\section{Introduction}

Ultra-fast four wave mixing parametric process in guided systems has been investigated in various configurations since it can exhibit a very large gain-bandwidth suitable for wide tunability and for the amplification of ultrashort pulses [1-5]. Ultra-short pulses with high peak power can be directly injected in hollow core fibers or capillaries that have high optical damage threshold [6]. When solid core fibers are the amplification medium, the ultra-short pulse is firstly chirped to decrease the peak power before amplification. In this case, the amplifier is often pumped by a strong quasi-continuous wave $(\mathrm{CW})$ with long (few ps or ns) pulse duration to temporally overlap with the stretched signal $[5,7-9]$. The parametric gain bandwidth defines the spectrum that can be amplified and thus the minimum pulse duration delivered by the amplifier. Some efforts have already been done to enhance the parametric bandwidth with different configurations, for instance, by employing an idlerband distributed fiber loss [10], a specific fiber with tailored properties $[11,12]$ or a single stretched short pulse which pumps a standard fiber [13]. Another possibility is to pump the fiber by two quasi-CW pulses $[14,15]$. This configuration exhibits very large spectral gain up to 300 $\mathrm{nm}$ between the two pump waves [16] which is a key feature for an ultra-fast amplifier [17]. However, this system

\footnotetext{
* Corresponding author:damien.bigourd@femto-st.fr
}

requires several pump lasers emitting spectrally shifted CWs that are synchronized with the stretched signal. In this manuscript, we are investigated the parametric process with two pump pulses with large bandwidth. There are several advantages to use chirped pulses instead of quasi-CWs. Firstly, the peak power can be much higher leading to a stronger gain. Secondly, the experimental set-up is easier to implement since the signal and pumps are in pulse mode as it is the case in most laser architectures delivering ultra-short pulses. Self-synchronized pump and signal pulses can be derived from an unique laser system by generating non linearity in fibers $[18,19]$, by selecting parts of the ultra-wide bandwidth of the signal [20] or by using a two color amplifier seeded by a continuum [21]. Each pulses are chirped with a standard grating based stretcher or compressor to pump the fiber. A chirped fiber Bragg grating can also be employed to realize a monolithic all fiber system. All the timing between the pulses can be adjusted only by delay stages without complex electronic devices. For example, in parametric amplifiers with nonlinear crystals, it is common to derive the pump and signal from one source $[20]$. Similar experimental setup can be implemented with our configuration and incorporated in a high power laser chain [22] or in a laser-matter experiment [23].

However, when pump chirped pulses are used, instantaneous frequencies are temporally separated and thus, a 
temporal distribution of the spectral gain is expected. In a previous work investigating parametric amplifier pumped by a single chirped pulse, we have observed and predicted the temporal distribution of the gain [13, 24]. Although the amplifier exhibits very large gain bandwidth, up to $36 \mathrm{THz}$, capable of amplifying sub-30 fs pulses [25], the amplified pulse needs to be firstly tailored such as the signal chirp matches the properties of the amplifier. In the case of the parametric amplifier pumped by two chirped pulses, this temporal distribution can be uniform, provided the chirps have opposite signs. The amplifier exhibits similar performances as the one pumped by two CWs. For other chirp relative signs, the amplifier properties become completely different and are not relevant for ultra-fast pulses. In the next section, an analytical model is presented to predict the behavior of the amplifier and several cases are discussed to highlight the role of the pump chirps and the group delay mismatch between all the pulses. In the third section, a chirped signal pulse with a very broad bandwidth is combined to demonstrate the amplification of ultra-short pulse with this amplifier.

\section{Analytical model of a parametric amplifier pumped by two chirped pulses}

\section{A. General principle and notations}

The general principle is based on a non-degenerate parametric process generally used with two continuous pump waves that provides very large gain bandwidth $[14,15]$. In the following, the pumps are in pulse mode and have relatively large bandwidth to allow high peak power and an experimental implementation from an unique laser source. The parametric amplifier pumped by two chirped pulses is described as a wave mixing of four pulses injected in a nonlinear fiber, including two pumps $\left(\mathrm{P}_{1}, \mathrm{P}_{2}\right)$ with the large bandwidth, a signal $\mathrm{S}$ and a generated idler I with a center angular frequencies $\omega_{p 10}$, $\omega_{p 20}, \omega_{s 0}$ and $\omega_{i 0}$, respectively (Fig. 1).

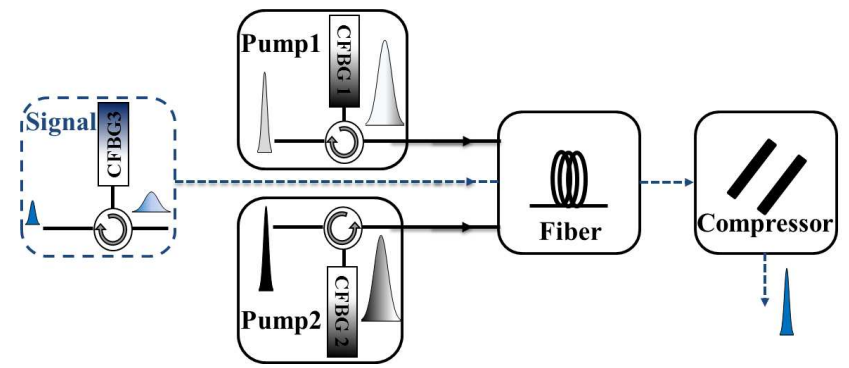

Fig. 1. Generic scheme of the parametric amplifier pumped by two chirped pulses. In this example, the pumps and signal are stretched by chirped fiber Bragg gratings (CFBG).

In order to amplify an ultra-short pulse with very large bandwidth, the signal is a stretched pulse and after amplification the chirp is compensated leading to an amplified and compressed ultra-short pulse (Fig. 1). Fig. 2 displays schematic spectrograms of the injected chirped pulses and introduces the notation required for

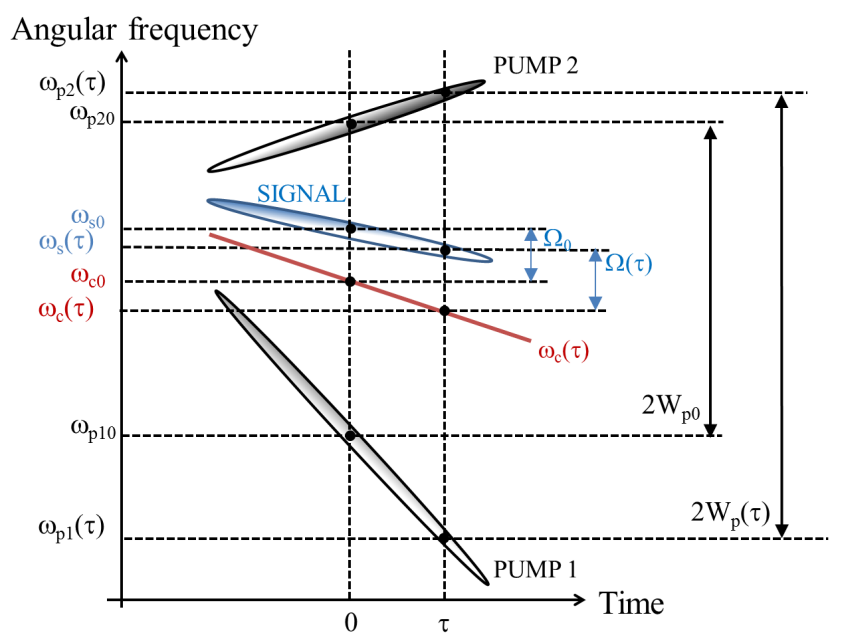

Fig. 2. Schematic spectrograms of the injected chirped pulses.

the model. The temporal phase applied to each pump pulse $\Phi_{p 10}(\tau), \Phi_{p 20}(\tau)$ induces a delay $\tau$ on each spectral component. The instantaneous angular frequencies are given by the time derivative of the phase. In the case of linear chirped pump pulses, the angular frequency shifts (Fig. 2) can be written as

$$
\begin{aligned}
& \omega_{p 1}(\tau)-\omega_{p 10}=\alpha_{p 1} \cdot \tau \\
& \omega_{p 2}(\tau)-\omega_{p 20}=\alpha_{p 2} \cdot \tau
\end{aligned}
$$

with $\alpha_{p 1, p 2}=-\partial \Phi_{p 1, p 2}(\tau) / \partial \tau$.

When pumping the amplifier with the two pulses owing relatively broad bands, two instantaneous pump frequencies generate an instantaneous parametric gain at the time $\tau$ with specific features.

Since FOPA is well described analytically with CWs [26], we consider the chirped pump pulses as the sum of independent monochromatic waves temporally separated by delays defined by their chirps. Accordingly, all the frequencies are derived from the central frequency $\omega_{c 0}=\frac{\omega_{p 20}+\omega_{p 10}}{2}$ at $\tau=0$. We defined the central frequency $\omega_{c}(\tau)$, half of the pump spectral spacing $W_{p}(\tau)$ and the angular frequency detuning between the signal and the central frequency $\Omega$ as (Fig. 2) :

$$
\begin{gathered}
\omega_{c}(\tau)=\frac{\omega_{p 2}(\tau)+\omega_{p 1}(\tau)}{2}=\omega_{c 0}+\frac{\alpha_{p 2}+\alpha_{p 1}}{2} \tau \\
W_{p}(\tau)=\frac{\omega_{p 2}(\tau)-\omega_{p 1}(\tau)}{2}=W_{p 0}+\frac{\alpha_{p 2}-\alpha_{p 1}}{2} \tau \\
\Omega(\tau)=2 \pi \times f(\tau)=\omega_{s}(\tau)-\omega_{c}(\tau)=\Omega_{0}-\frac{\alpha_{p 2}+\alpha_{p 1}}{2} \tau
\end{gathered}
$$


with $W_{p 0}=W_{p}(\tau=0)$ and $\Omega_{0}=\Omega(\tau=0)$.

The dispersion coefficients involved in the derivation are evaluated at the central frequency $\omega_{c}\left(\beta_{2 c}, \beta_{3 c}, \beta_{4 c 0}\right)$ and therefore evolved with time from those at $\tau=0$, $\left(\beta_{2 c 0}, \beta_{3 c 0}, \beta_{4 c 0}\right)$ :

$$
\begin{gathered}
\beta_{2 c}(\tau)=\beta_{2 c 0}+\beta_{3 c} \frac{\alpha_{p 2}+\alpha_{p 1}}{2} \tau+\frac{\beta_{4 c 0}}{2}\left(\frac{\alpha_{p 2}+\alpha_{p 1}}{2} \tau\right)^{2} \\
\beta_{3 c}(\tau)=\beta_{3 c 0}+\beta_{4 c 0} \frac{\alpha_{p 2}+\alpha_{p 1}}{2} \tau
\end{gathered}
$$

\section{B. Description of the model}

A previous analytical model has been developed to describe the parametric amplification when a fiber is pumped by a single chirped pulse [24]. In the following, we present the modification of the model to predict the behavior of parametric amplifier pumped by two chirped pump pulses with the relevant parameters (Eq. 3-7) in the appropriate frame. In order to calculate the amplifier properties, a CW signal is preferentially injected and tuned over the full gain bandwidth; i.e $\omega_{s}(\tau)=\omega_{s 0}$. All the waves have the same polarization states. No Raman scattering contribution is considered and the pumps are not depleted; i.e the pump powers are much stronger than all the other waves. From Eq. 3-7, the appropriate angular frequencies and the dispersion parameters evolve with time and this variation can impact directly the temporal distribution of the spectral gain. When the two pump pulses are injected into a loss-less fiber with a nonlinear coefficient $\gamma$ along the $z$ propagation direction, the signal and idler electric field can be calculated from the equations obtained with two CW pump waves [26]. $P_{p 1}$ and $P_{p 2}$ are the instantaneous power of the two pumps. For sake of clarity, the time dependency of all parameters is not written in the following equations. By including the previous variables (Eq. 3-7), we obtained

$S\left(z, \Omega_{0}, \tau\right)=e^{j\left(\frac{3}{2} \gamma P_{p t}+\frac{1}{2} \beta_{2 c} \omega_{p}^{2}+\frac{1}{6} \beta_{3 c} \Omega^{3}+\frac{1}{24} \beta_{4 c} \omega_{p}^{4}\right) z}\left\{S_{0}\left(\Omega_{0}\right)\left(\cosh (g z)+j \frac{\kappa}{2 g} \sinh (g z)\right)+j I_{0}^{*}\left(-\Omega_{0}\right) \frac{2 \gamma \sqrt{P_{p 1} P_{p 2}}}{g} \sinh (g z)\right\}$

$$
I^{*}\left(z,-\Omega_{0}, \tau\right)=e^{-j\left(\frac{3}{2} \gamma P_{p t}+\frac{1}{2} \beta_{2 c} \omega_{p}^{2}-\frac{1}{6} \beta_{3 c} \Omega^{3}+\frac{1}{24} \beta_{4 c} \omega_{p}^{4}\right) z}\left\{I_{0}^{*}\left(-\Omega_{0}\right)\left(\cosh (g z)-j \frac{\kappa}{2 g} \sinh (g z)\right)-j S_{0}\left(\Omega_{0}\right) \frac{2 \gamma \sqrt{P_{p 1} P_{p 2}}}{g} \sinh (g z)\right\}
$$

with $\mathrm{S}_{0}$ and $\mathrm{I}_{0}$ the input signal and idler electric field, $P_{p t}=P_{p 1}+P_{p 2}$ the total pump power, and $g$ the parametric gain coefficient given by

$$
g=g\left(\Omega_{0}, \tau\right)=\sqrt{4 \gamma^{2} P_{p 1} P_{p 2}-\frac{\kappa\left(\Omega_{0}, \tau\right)^{2}}{4}}
$$

The total phase matching parameter is $\kappa\left(\Omega_{0}, \tau\right)=$ $\Delta \beta\left(\Omega_{0}, \tau\right)+\gamma P_{p t}$ where $\Delta \beta$ is the linear phase matching parameter given by

$$
\Delta \beta\left(\Omega_{0}, \tau\right)=\beta_{2 c}\left[\Omega^{2}-W_{p}^{2}\right]+\frac{\beta_{4 c 0}}{12}\left[\Omega^{4}-W_{p}^{4}\right]
$$

From Eq. 11, we deduce that the linear phase matching parameters depends strongly on the two chirp values and their signs leading to a direct impact on the spectrotemporal distribution of the gain. Two cases are detailed in the following where the two chirps have identical or opposite signs. For sake of simplicity, we do not take into account $\beta_{4 c 0}$ in the following derivation. We also assume the pump bandwidths are much smaller than the spectral spacing between the two pumps and the signal angular frequency detuning $\left(\alpha_{p 1, p 2} \cdot \tau<<W_{p 0}\right.$, $\left.\Omega_{0}\right)$ and $\Omega_{0}$ is away from $W_{p 0}$. The relative variation of the phase matching from the value at $\tau=0$ is calculated as $\delta=\frac{\Delta \beta\left(\Omega_{0}, \tau\right)-\Delta \beta\left(\Omega_{0} \tau=0\right)}{\Delta \beta\left(\Omega_{0}, \tau=0\right)}$. We can show that $\delta$ can be written for $\alpha_{p 1}=\alpha_{p 2}=\alpha\left(\operatorname{case} \delta_{1}\right)$ and $\alpha_{p 1}=\alpha_{p 2}=-\alpha$ $\left(\right.$ case $\left.\delta_{2}\right)$ as :

$$
\begin{gathered}
\delta_{1}\left(\alpha_{p 1}=\alpha_{p 2}=\alpha_{p}\right) \approx \frac{\beta_{3 c 0}}{\beta_{2 c 0}} \alpha_{p} \tau \\
\delta_{2}\left(\alpha_{p 1}=-\alpha_{p 2}=-\alpha_{p}\right) \approx 0
\end{gathered}
$$

When the two chirps have opposite values, the linear phase matching is uniform for a wide range of signal angular frequency $\left(\Omega_{0}\right)$ provided the bandwidth is relatively small compared to the pump frequency spacing (Eq. 13). Therefore, we can expect a flat spectrotemporal distribution of the gain during the pump pulse durations while the spectral gain will change over the time for identical chirp values (Eq. 12). 


\section{C. Numerical simulations performed for the vali- dation of the model}

In order to validate the analytical model, realistic numerical simulations have been conducted by integrating the nonlinear Schrödinger equation (NLSE) along the propagation $z$

$$
i \frac{\partial \psi}{\partial z}-\frac{\beta_{2}(z)}{2} \frac{\partial^{2} \psi}{\partial t^{2}}-i \frac{\beta_{3}}{6} \frac{\partial^{3} \psi}{\partial t^{3}}+\frac{\beta_{4}}{24} \frac{\partial^{4} \psi}{\partial t^{4}}+\gamma|\psi|^{2} \psi=0
$$

describing the evolution of the slowly varying total electric field $\psi$ in an optical fiber. Eq. 14 has been solved with the standard split-step Fourier. The temporal resolution is $8 \mathrm{fs}$ and the number of points is $2^{18}$. The input electric field includes a weak random initial condition that mimics quantum fluctuations, the two chirped pump pulses and a weak CW signal whose frequency is tuned over the full gain bandwidth. After propagation, the signal is selected by a spectral filter and the gain is calculated in the temporal domain for each frequency to map its distribution.

\section{D. Discussion of selected cases}

To illustrate the discussion, we consider several cases with parameters close to those used in previous experiments [13] and numerical analysis [24]. The two pump pulses have a duration of $900 \mathrm{ps}$ full width at half maximum (FWHM) with a super-Gaussian temporal profile. The central angular frequencies are $\pm(2 \pi) \times 15 \mathrm{THz}$ $\left(\omega_{c 0}=0\right)$. The peak powers are $P_{p 1}=P_{p 2}=10 \mathrm{~W}$ and the temporal chirp of pulse 1 is $\alpha_{p 1}=-(2 \pi) \times 2.25$ $\mathrm{THz} / \mathrm{ns}$ while $\alpha_{p 2}$ varies. Therefore, the spectral bandwidth of pulse 1 is $(2 \pi) \times 2.02 \mathrm{THz}(\mathrm{FWHM})$ while it changes for the second pulse. For example, when $\alpha_{p 2}=0$, the wave is quasi-monochromatic. The pumps are injected in a fiber owing a nonlinear coefficient $\gamma=35$ $\mathrm{W}^{-1} \mathrm{~km}^{-1}$ and $\beta_{20}, \beta_{30}, \beta_{40}$ equal to $1 \times 10^{-28} \mathrm{~s}^{2} / \mathrm{m}$, $1 \times 10^{-40} \mathrm{~s}^{3} / \mathrm{m},-1 \times 10^{-55} \mathrm{~s}^{4} / \mathrm{m}$, respectively. The propagation distance is $L=10 \mathrm{~m}$.

The gain profile in the anti-Stokes side is calculated at each time from Eq. 8 and Eq. 9. Gain maps representing the spectral gains as a function of time $\tau$ are displayed in Fig. 3 for three cases in which $\alpha_{p 2}=-\alpha_{p 1}$ (Fig. 3.a), $\alpha_{p 2}=+\alpha_{p 1}$ (Fig. 3.b) and $\alpha_{p 2}=0$ (Fig. 3.c). Fig. 3.g$\mathrm{h}-\mathrm{i}$ (solid lines) represent the instantaneous gain profiles at $\tau=0$ (white dashed lines in the maps). Fig. 3.j shows the temporal distribution of the gain at the central frequency $(f=0 \mathrm{THz})$ for the three cases : $\alpha_{p 2}=-\alpha_{p 1}$ (blue line), $\alpha_{p 2}=+\alpha_{p 1}$ (red line) and $\alpha_{p 2}=0$ (green line). The temporal distributions of the gain change considerably for the three cases. For $\alpha_{p 2}=-\alpha_{p 1}$, the spectral gain is uniform at each $\tau$ since the variation of the phase matching is weak during the pump pulse duration as expected from $\delta_{2}$ (Eq. 13). The maximum gain is $\sim 55 d B$ and varies by less than $8 \mathrm{~dB}$ from 0 to $22 \mathrm{THz}$ (Fig. 3.g). The ripples and the number of extrema depend on the amplifier properties as in the case
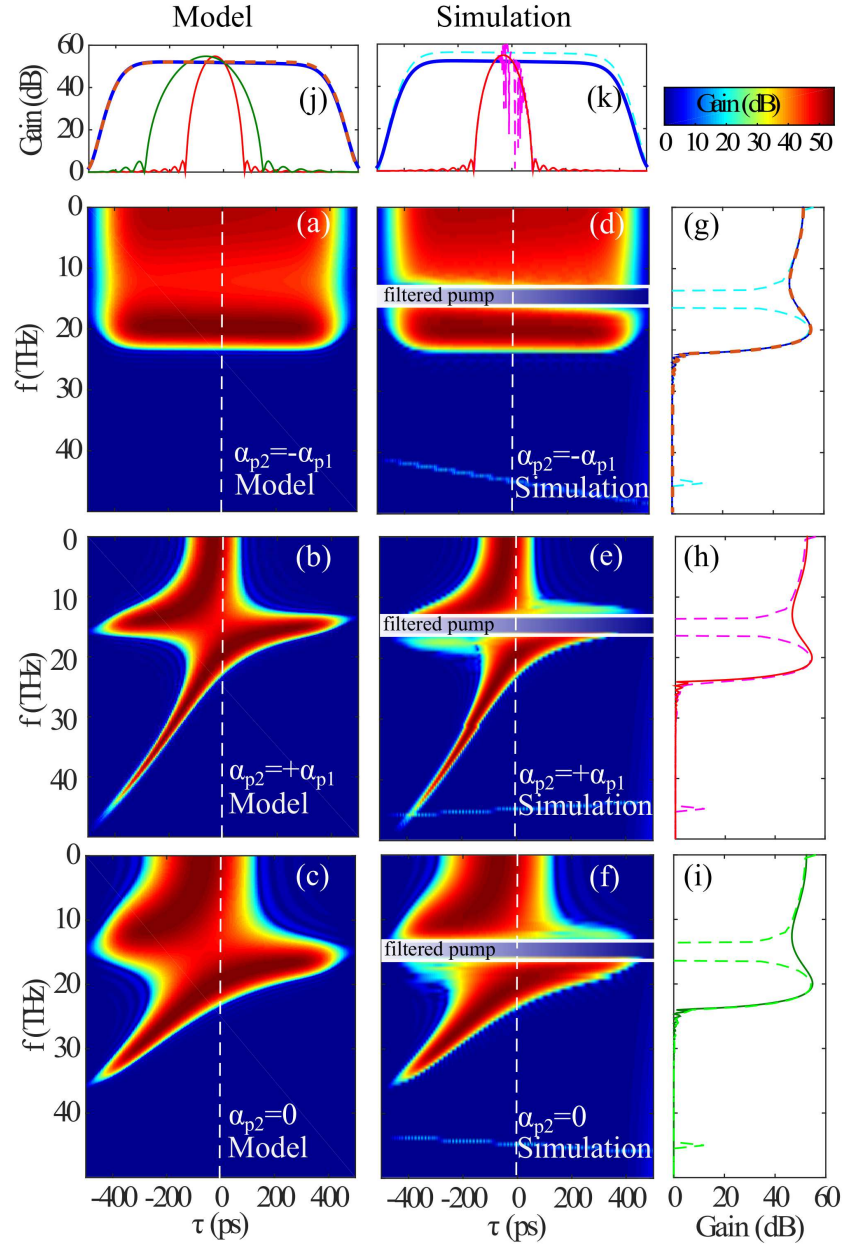

Fig. 3. Gain maps calculated in the Anti-Stokes side from the analytical model and the NLSE simulation for $\alpha_{p 2}=-\alpha_{p 1}$ (a-d), $\alpha_{p 2}=+\alpha_{p 1}$ (b-e) and $\alpha_{p 2}=0$ (c-f). The spectral gain at $\tau=0 \mathrm{ps}$ for $\alpha_{p 2}=-\alpha_{p 1}, \alpha_{p 2}=+\alpha_{p 1}$ and $\alpha_{p 2}=0$ are shown in (g), (h) and (i). The dashed and solid lines correspond to the results obtained with the simulation and the model, respectively. The gain as a function of $\tau$ at $\mathrm{f}=0$ $\mathrm{THz}$ are displayed in (j) for $\alpha_{p 2}=-\alpha_{p 1}$ (blue line), $\alpha_{p 2}=$ $+\alpha_{p 1}$ (red line) and $\alpha_{p 2}=0$ (green line). The dashed brown line is calculated for two CW pumps. The blue and red lines are reproduced in $(\mathrm{k})$. The corresponding curves obtained with the NLSE simulation are also shown in (k) $\left(\alpha_{p 2}=-\alpha_{p 1}\right.$ (cyan line), $\alpha_{p 2}=+\alpha_{p 1}$ (magenta line))

of an amplifier pumped by two CWs. The flatness is improved for reduced $\beta_{40}$ value and higher $\gamma P_{p t}$ [27]. The extrema between the two pumps are located at $0 \mathrm{THz}$ (maximum) and $\pm 12.3 \mathrm{THz}$ (minimum) in good agreement with the expected location, $\pm \frac{1}{2 \pi} \sqrt{\frac{-6 \beta_{2 c 0}}{\beta_{4} c 0}}$ derived from the case with two CW pumps [27]. We checked that the spectral distribution of the gain is nearly the same as the one calculated for two continuous pump waves. The corresponding curve displaying the gain evolutions as respect to the time at $f=0 \mathrm{THz}$ (Fig. 3.j) and to the frequency at $\tau=0$ (Fig. 3.g) are similar to the one ob- 
tained with the chirped pulses. The variations are not observed on these scales : the gain is unchanged over the full temporal profile for the $\mathrm{CW}$ while it changes by $1 \mathrm{~dB}$ in the case of the chirped pulses. For the case where $\alpha_{p 2}=+\alpha_{p 1}$, the spectral gain changes strongly with time (Fig. 3.b) as expected from Eq. 12. Similarly, for $\alpha_{p 2}=0$, the map is not uniform (Fig. 3.c) mainly due to the variation of the dispersion over the pump bandwidth. For $\alpha_{p 2}=+\alpha_{p 1}, \beta_{2 c}$ changes from $-6 \times 10^{-28}$ to $+8 \times 10^{-28} \mathrm{~s}^{2} / \mathrm{m}$ when the pump frequency is swept during the pulse duration leading to a significant change of the gain profile. The maximum gain is similar to the previous case but it extends to higher frequency up to $\sim 40 \mathrm{THz}$ at $\tau \sim-300 \mathrm{ps}$ (for $\alpha_{p 2}=+\alpha_{p 1}$ ) and $\sim 32 \mathrm{THz}$ at $\tau \sim-350$ ps (for $\alpha_{p 2}=0$ ). However, each frequency is amplified at a given time making the amplifier less convenient than the previous case $\left(\alpha_{p 2}=-\alpha_{p 1}\right)$. For example, the maximum gain at $f=0 \mathrm{THz}$ only occurs at $\sim-30 \mathrm{ps}$ and $-70 \mathrm{ps}$ for $\alpha_{p 2}=+\alpha_{p 1}$ (Fig. 3.a-red line) and $\alpha_{p 2}=0$ (Fig. 3.a-green line), respectively. In addition, the temporal width of the gain depends on $\tau$. Consequently, the efficient amplification of a chirped signal pulse with large bandwidth will require to match the signal chirp with the temporal distribution of the gain. Numerical simulations have been performed with the same parameters as those used in the analytical model. The temporal distributions of the spectral gain are displayed in Fig. 3.d-e-f next to the maps obtained with the analytical model together with the gains at $\tau=0$ (g-cyan dashed line, h-magenta dashed line,i-light green dashed line). Excellent agreements are obtained for the three discussed cases confirming the correct behavior of the analytical model. Weak additional informations are provided by the numerical simulations. Beyond $\sim 40 \mathrm{THz}$, weak degenerated four wave mixings between the pumps appear. In addition, the contribution of the idler can be observed with the simulation. For example, the gain at $f=0 \mathrm{THz}$ as respect to the time is displayed in Fig. 3.k for $\alpha_{p 2}=-\alpha_{p 1}$ (cyan dashed line) and $\alpha_{p 2}=+\alpha_{p 1}$ (magenta line) together with the corresponding curves obtained from the analytical model (blue and red solid lines). In fact, the idler generated and filtered at $f=0 \mathrm{THz}$ interferes with the signal wave at the same frequency. However, the frequency of the generated idler changes with time during the interaction with the other pulses. From the photon energy conservation, the frequency is given by $f_{i}=\left(\alpha_{p 1}+\alpha_{p 2}\right) \tau /(2 \pi)-f=\left(\alpha_{p 1}+\alpha_{p 2}\right) \tau /(2 \pi)$. When $\alpha_{p 2}=-\alpha_{p 1}$, the idler at $0 \mathrm{THz}$ is generated for each $\tau$ value simultaneously with the signal at $f=0 \mathrm{THz}$. Therefore, no spectral modulation from interferences is observed in the time domain. The gain is $3 \mathrm{~dB}$ higher (Fig. 3.k-cyan dashed line) than the one obtained with the analytical model since both signal and idler contribute to the output at $f=0 \mathrm{THz}$. For $\alpha_{p 2}=\alpha_{p 1}$, the idler at $f=0 \mathrm{THz}$ is generated only around $\tau=0$ and interferes with the signal leading to temporal modulation (Fig. 3.k-magenta line).

\section{E. Influence of the group delay mismatch}

During the propagation, each pulses propagate with different group velocity and the monochromatic signal experiences a range of pump frequencies from the two pulses along the propagation. The group delay (GD) is given by the frequency derivative of the phase. During propagation, the two pumps have been delayed by

$$
\tau_{1,2}=\left(\mp \beta_{2 c} W_{p}+\frac{\beta_{3 c}}{2} W_{p}^{2} \mp \frac{\beta_{4 c 0}}{12} W_{p}^{3}\right) z
$$

The GD of the signal is calculated by assuming that the amplifier is phase matched and we only use the exponential term in Eq. 8. The group delay of the signal is

$$
\tau_{s}=\frac{\beta_{3 c}}{2}\left(\Omega_{0}-\frac{\alpha_{p 1}+\alpha_{p 2}}{2} \tau\right)^{2} z
$$

The GD effect has been included in the model by calculating step by step the Stokes and anti-Stokes amplitudes (Eq. 8 and Eq. 9) along the fiber. Delays $T_{1,2}=\tau_{s}-\tau_{1,2}$ have been added at each step shifting the parameters $\omega_{c}, W_{p}$ (Eq. 3 and Eq. 4) by $\Delta \omega_{c}=\frac{\alpha_{p 2} T_{2}+\alpha_{p 1} T_{1}}{2}$ and $\Delta W_{p}=\frac{\alpha_{p 2} T_{2}-\alpha_{p 1} T_{1}}{2}$, respectively. These equations clearly show that the effect of the GD will depend on the value of both chirps. Gain maps are displayed in Fig. 4.a-b. for an amplifier pumped by two chirped pulses but with $P_{p 1}=P_{p 2}=1 \mathrm{~W}$ with $L=100 \mathrm{~m}$ for $\alpha_{p 2}=\mp \alpha_{p 1}$.

The spectral gain has ripples with much higher modulation depth than in the previous case since they increase with lower pump power, as observed in a two CW pump amplifier [27]. For comparison, the same map has been analytically calculated with no GD mismatch between the pulses (Fig. 4.c-d). We can notice that the effect of the GD is negligible when $\alpha_{p 2}=-\alpha_{p 1}$ while the gain decreases for frequency away from the pump when $\alpha_{p 2}=+\alpha_{p 1}$. For example, for $\Omega_{0}=0 \mathrm{THz}$ and $\tau=0 \mathrm{ps}$, $\tau_{1,2} \sim 44$ ps and $\tau_{s}=0$ in both cases. However, $\Delta \omega_{c}$ and $\Delta W_{p}$ are equals to 0 and $\sim-0.7 \mathrm{THz}\left(\alpha_{p 2}=-\alpha_{p 1}\right)$ while they are equal to $\sim-0.7 \mathrm{THz}$ and $0 \mathrm{THz}\left(\alpha_{p 2}=+\alpha_{p 1}\right)$. In the latter case, the important variation of $\omega_{c}$ in comparison to the central frequency at $L=0 \mathrm{~m}\left(\omega_{c}=0\right)$ leads to a drop of the gain. The fiber length should be short to limit the GD effect. For example, the GD does not impact the gain shape when the fiber length is decreased to $\mathrm{L}=10 \mathrm{~m}$. Fig. 4.e-f are the corresponding maps obtained from the NLSE simulation and excellent agreements are obtained with the analytical model when the GD effect is included.

\section{F. Impact of the pump chirps on a chirped signal pulse}

A chirped signal has a quasi-linear temporal distribution of the spectrum. Each frequency needs to be amplified 

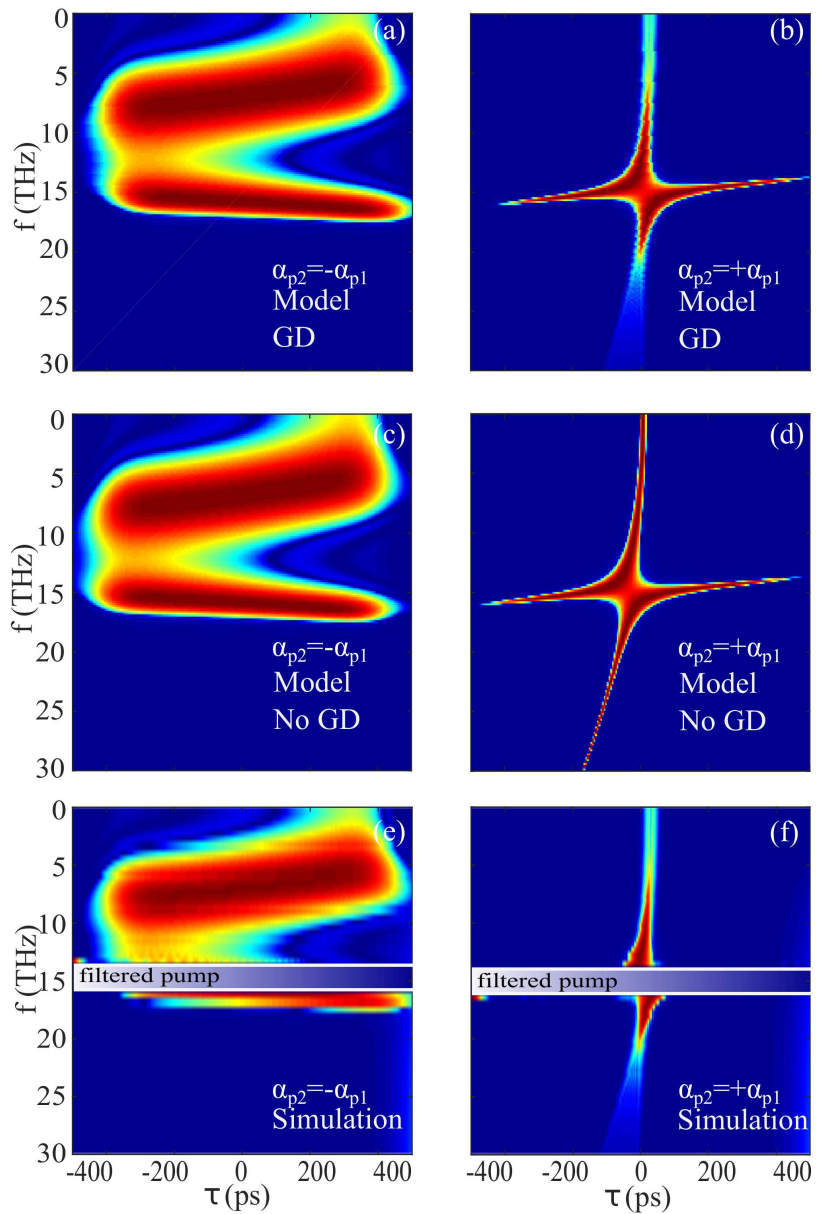

Fig. 4. Gain maps calculated in the Anti-Stokes side from the analytical model $\alpha_{p 2}=-\alpha_{p 1}$ (a) and $\alpha_{p 2}=+\alpha_{p 1}$ (b) including the GD effect. (c,d) are the maps when the GD is neglected and $(e, f)$ are the corresponding gain obtained with the NLSE simulation. The colorbar is the same as in Fig.3.

at the appropriate time. However, the spectral gain of the amplifier varies strongly with time in several cases making the parametric amplifier not convenient for the amplification of this stretched signal pulse; i.e the properties of the amplifier need to be engineered. For efficient amplification of a large bandwidth, the spectrotemporal distribution of the signal needs to match the gain map. This optimization is similar to the case of a single chirped pump [25] and some suitable configurations can be found if the signal chirp is preliminary adjusted. However, when the two pump chirps have opposite values, the gain profile does not change with time. This means that any chirped signal with the correct spectral bandwidth are expected to be amplified. Firstly, we consider a signal with a linear chirp $\alpha_{s}=$ $2 \pi \times 50 \mathrm{THz} / \mathrm{ns}$ corresponding to a frequency sweep from $-20 \mathrm{THz}$ to $+20 \mathrm{THz}$ when $\tau$ changes from -400 ps to +400 ps. Fig. 5 displays the instantaneous gain for selected signal frequencies when $\alpha_{p 1}$ is fixed and $\alpha_{p 2}$ is varied. The gain changes significantly excepted for $f=0 \mathrm{THz}(\tau=0)$ where the gain is constant since the dispersion does not vary with the chirp at this time. For $\alpha_{p 2}=-\alpha_{p 1}$, all the selected frequencies have similar gain $(\sim 49 \mathrm{~dB} \pm 3 \mathrm{~dB})$ making this configuration suitable for the amplification of large bandwidth. For $f= \pm 20$ $\mathrm{THz}$, the corresponding time is $\pm 400 \mathrm{ps}$ where the pump power decreases by $\sim 20 \%$ leading to a gain drop.

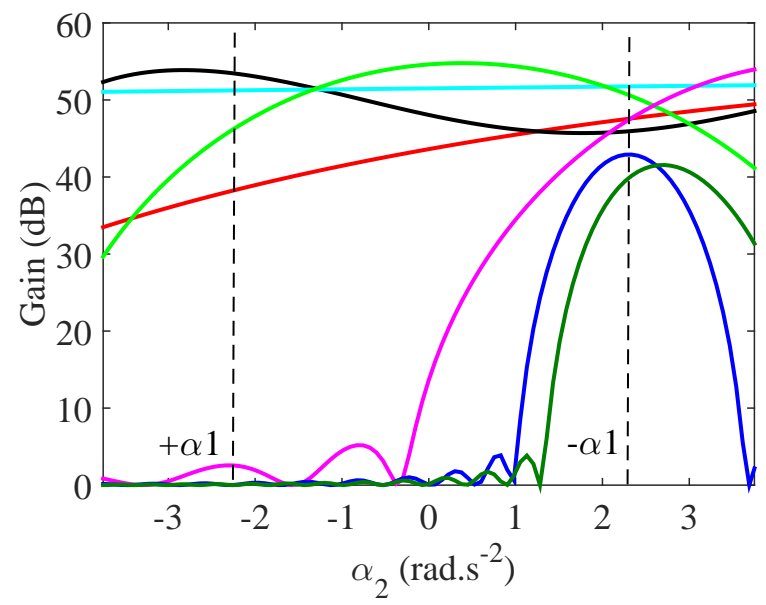

Fig. 5. Gain as a function of $\alpha_{p 2}$ for $f=-20 \mathrm{THz}$ (blue line),$10 \mathrm{THz}$ (red line), -7 THz (light green line), $0 \mathrm{THz}$ (cyan line), $+7 \mathrm{THz}$ (magenta line), $+10 \mathrm{THz}$ (black line), +20 $\mathrm{THz}$ (dark green) when a signal is injected with a chirp $\alpha_{s}=2 \pi \times 50 \mathrm{THz} / \mathrm{ns} . \alpha_{p 1}=-(2 \pi) \times 2.25 \mathrm{THz} / \mathrm{ns}$.

The gain of a chirped signal pulse is displayed in Fig. 6 for $\alpha_{s}=2 \pi \times 50 \mathrm{THz} / \mathrm{ns}, \alpha_{s}=2 \pi \times 100 \mathrm{THz} / \mathrm{ns}$, $\alpha_{s}=2 \pi \times 200 \mathrm{THz} / \mathrm{ns}$, in the frequency (Fig. 6.a-c) and temporal (Fig. 6.b-d) domains.

For $\alpha_{p 2}=+\alpha_{p 1}$ (Fig. 6.c-d), the gain changes considerably and is structured when the signal chirp varies. However, the amplification can occur at higher frequency for selected chirp in which the spectro-temporal distribution matches the gain map at high frequencies (Fig. 3.b). For example, for $\alpha_{s}=2 \pi \times 200 \mathrm{THz} / \mathrm{ns}$ (green curves), $30 \mathrm{THz}$ occurs at $\tau=-150 \mathrm{ps}$ and therefore can be amplified (Fig. 6.d). For $\alpha_{p 1}=-\alpha_{p 2}$ (Fig. 6.a,b), the spectral gain does not change for any signal chirp value. For $\alpha_{s}=2 \pi \times 50 \mathrm{THz} / \mathrm{ns}$, the frequencies beyond $\sim 20 \mathrm{THz}$ are amplified by the edge of the pump leading to a decrease of the gain, as already observed in Fig. 5. By adjusting the signal chirp, the signal pulse duration increases and the instantaneous spectral gain occurs at other times (Fig. 6.b). Accordingly, the amplification of a chirped signal pulse with large bandwidth is very flexible when the pump chirps have opposite signs. The choice of the signal chirp is not defined by the properties of the amplifier and therefore this configuration is very promising for the design of a laser architecture. 

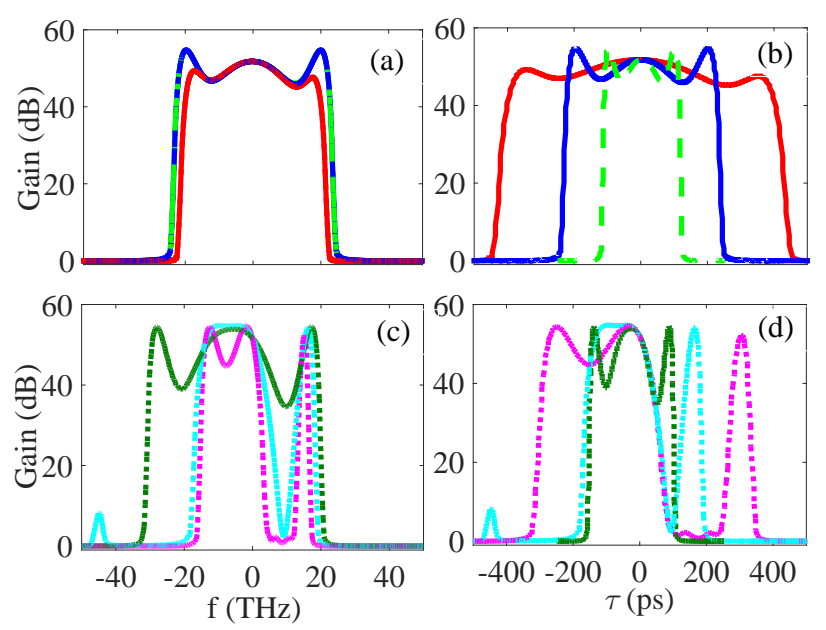

Fig. 6. Gain as a function of the signal frequency (left column) and the time (right column) for $\alpha_{p 2}=-\alpha_{p 1}$ (top line) and $\alpha_{p 2}=+\alpha_{p 1}$ (bottom line) for $\alpha_{s}=2 \pi \times 50 \mathrm{THz} / \mathrm{ns}$ (red lines), $\alpha_{s}=2 \pi \times 100 \mathrm{THz} / \mathrm{ns}$ (blue lines), $\alpha_{s}=2 \pi \times 200 \mathrm{THz} / \mathrm{ns}$ (green curves).

\section{Ultra-short pulse amplification with two chirped pump pulses}

To confirm the possibility to amplify ultra-short pulses with an amplifier pumped by two chirped pulses, numerical simulations have been performed as in Section 2.B. The signal has a Fourier transform limited pulse duration equals to 50 fs with a Gaussian profile corresponding to a spectral bandwidth of $\sim 8.76 \mathrm{THz}$ (FWHM). The pulse duration is increased to 300 ps with a standard stretcher to match the pulse duration of both pumps and to decrease the peak power. The signal spectrum is centered between the two pumps $\left(\Omega_{0}=0\right.$ $\mathrm{THz}$ ) to take benefit from the full gain bandwidth. The spectrogram of the input pulse together with the temporal and spectral shapes are shown in Fig. 7. The black lines display the central frequencies of the two pumps. The fiber length $L=6.5 \mathrm{~m}$ has been chosen to amplify the signal in an unsaturated regime. A spectrogram of the total electric field is calculated at the output of the fiber. The two configurations of the

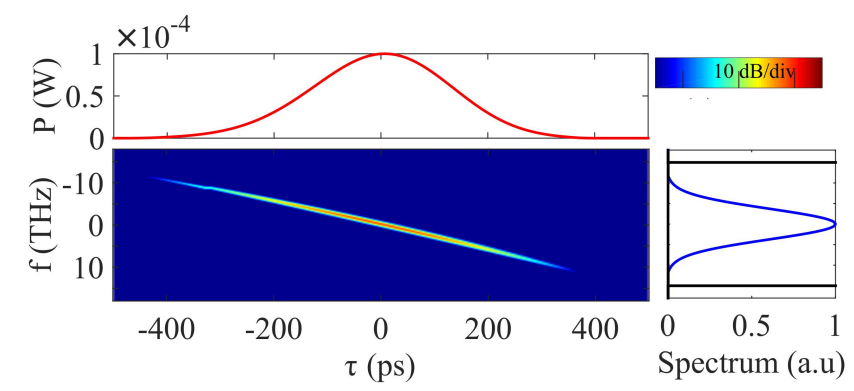

Fig. 7. Spectrogram, temporal and spectral profiles of the input signal. pumps are considered; i.e $\alpha_{p 1}=\alpha_{p 2}$ (Fig.8.a) and $\alpha_{p 1}=-$ $\alpha_{p 2}$ (Fig. 8.b). They are compared to the case where two quasi-CW pumps are used for the amplification (Fig. 8.c). In the three cases, the spectrograms show a "X" shape as observed in [17] in the case of two CW pumps. At each time, the signal is amplified at its instantaneous frequency while the idler is generated at $\omega_{i}=\omega_{p 1}+\omega_{p 2}-\omega_{s}$. The contribution of both pump chirps and bandwidths are actually weak and impact marginally the idler. Thus, the idler frequencies are generated with an opposite temporal distribution as respect to the signal. The combined contribution of the signal and idler leads to the shape observed on the spectrogram. The spectrum contains the same components generated at different time leading to interferences of the total electric field in the spectral and temporal domain. The results obtained with the opposite pump chirps (Fig. 8.b) are the same as the ones obtained with two CW pump waves (Fig. 8.c) confirming the suitable behavior of the dual chirped pump pulse amplifiers. The peak gain is $\sim 35 \mathrm{~dB}$ and the amplified bandwidth has not been significantly narrowed during the amplification. On the contrary, the signal amplified by two pumps with the same chirp values exhibits a narrowed spectrum with a shift toward lower frequencies (Fig. 8.a).

At the output of the fiber, the two pumps have been filtered out to keep only the signal and idler pulses. Then, a standard compressor has been optimized to reduce the pulse duration of the signal near the Fourier transform limit (Fig. 9). The compressor has two gratings owing 1200 grooves $/ \mathrm{mm}$. The angle and the grating separation have been adjusted to best compress the signal pulse in each case. They are $\sim 81^{\circ}$ and $0.9 \mathrm{~m}$, respectively. For opposite value of the pump chirps, the compressed pulse is $\sim 60$ fs (Fig. 9, blue line) near the input pulse duration (Fig. 9, black line). The compressed pulse amplified by the two CW pumps has the same pulse duration (Fig. 9, red line). We also checked that shorter pulse duration can be reached in the saturation regime as in [17]. For example, the compressed pulse duration is $50 \mathrm{fs}$ when the signal is amplified in a $L=7 \mathrm{~m}$ long fiber. When the two pumps have the same chirp value, the pulse duration is increased to $\sim 200$ fs (Fig. 9, magenta line) as expected from its gain narrowed spectrum (Fig. 8.a).

Fig. 9.b shows an enlargement of the compressed pulses in the few hundred ps window. The long pulses correspond to the generated idler. As it has the same spectrum, it cannot be filtered and also experiences the phase from the compressor device. Therefore, as the idler has an opposite chirp from the signal, it is stretched further by the compressor to twice the input pulse duration of the signal; i.e $600 \mathrm{ps}$. In this case, the temporal contrast is $\sim \mathbf{3 8} \mathbf{d B}$. For $\alpha_{p 1}=+\alpha_{p 2}$, the idler pulse duration is shorter than the one in the other cases because of the gain narrowed spectrum and the contrast 

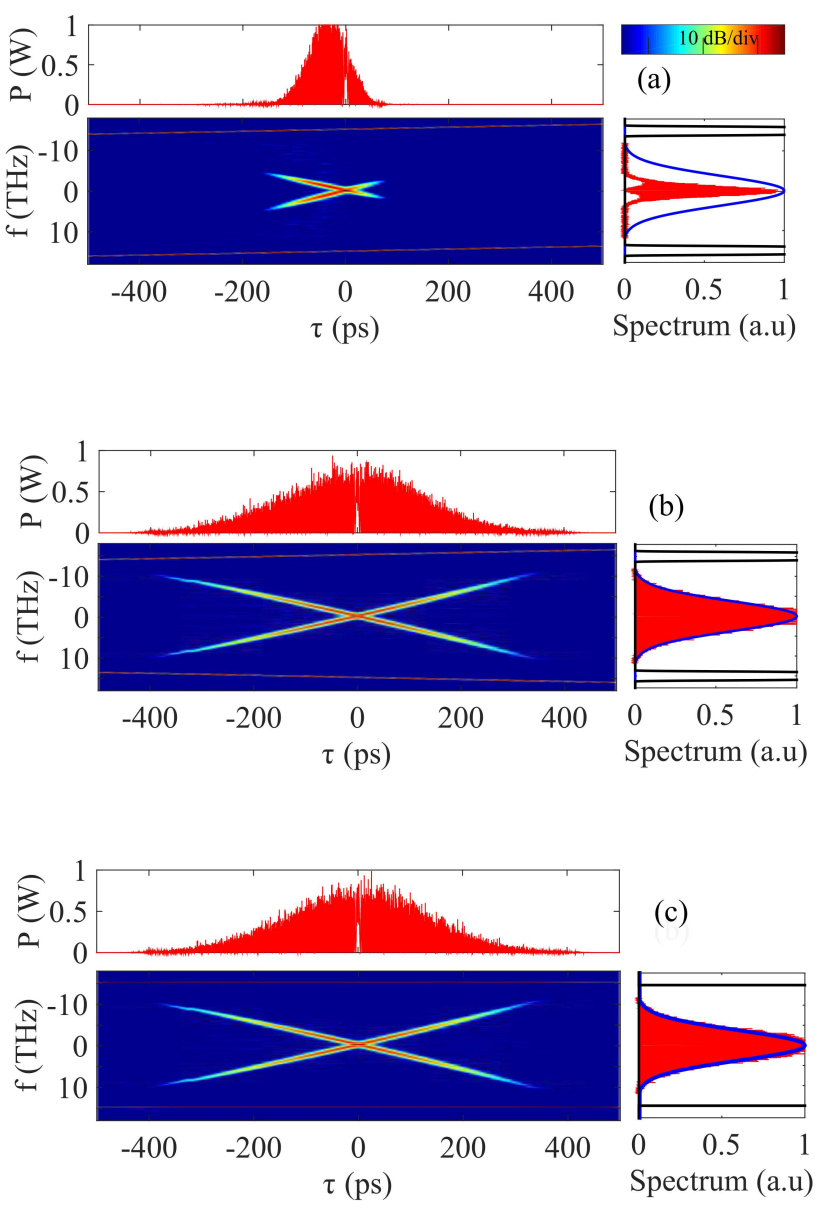

Fig. 8. Spectrogram, temporal and spectral profiles of the output electric field for two pumps with the same (a) and opposite chirps (b) and with two quasi-CW pumps (c).

is $\sim 30 \mathrm{~dB}$.

We have also calculated the optical to optical conversion efficiency defined as the energies of the signal and idler divided by the input energy of both pumps. We found that the efficiency reaches $60 \%$ at saturation (for $L=15 \mathrm{~m}$ ) when two CWs or two pulses with opposite chirps pump the fiber while it is only $15 \%$ when the pulses have the same chirps.

\section{Conclusion}

We have analytically and numerically investigated optical parametric amplifier pumped by two chirped pump pulses with relatively large bandwidth. In particular, we highlighted that the properties of the amplifier depends strongly on the relative chirp values and a uniform spectro-temporal gain can be obtained when the two chirps have opposite values. We also demonstrate the possibility to amplify ultra-short pulses with this scheme particularly suitable for laser architectures that deliver several ultra-short pulses.

Acknowledgments Computations have been per-
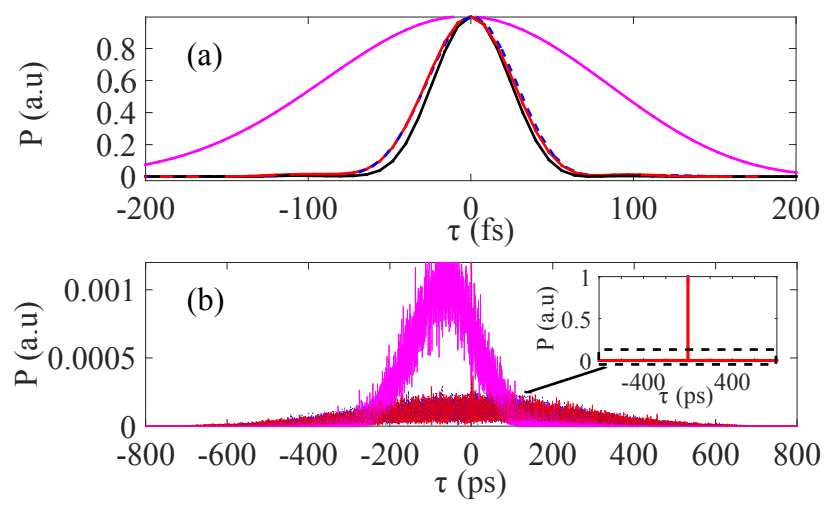

Fig. 9. (a)Temporal profile of the input pulse (black line) and the compressed pulse for two CW pumps (red line), two pumps with the opposite (blue line) and the same chirps (magenta line). (b) Observation of the pedestals in the larger time window. The dashed square corresponds to the zone of enlargement

formed on the supercomputer facilities of the Mésocentre de calcul de Franche Comté. This work was partly supported by the Labex ACTION program (ANR-11LABX-01-01).

\section{References}

[1] D. Faccio, A. Gun, P. K. Bates, O. Chalus, J. Biegert, "Optical amplification in the near-infrared in gas-filled hollow-core fibers," Opt. Lett. 34, 2918 (2009).

[2] T. Flory, P. Malevich, A. Puglys, A. Voronin, A.M. Zheltikov, A. Baltuska, "Kerr-gated generation of fewcycle mid-IR pulses in a four-wave mixing parametric amplifier", Advanced Solid State Lasers, ATu1A.4 (2014).

[3] D. Nodop, C. Jauregui, D. Schimpf, J. Limpert, A. Tunnermann, "Efficient high-power generation of visible and mid-infrared light by degenerate four-wave-mixing in a large-mode-area photonic-crystal fiber", Opt. Lett. 34, 3499 (2009).

[4] M. Hanna, F. Druon, P. Georges, "Fiber optical parametric chirped pulse amplification in the femtosecond regime," Opt. Express 14, 2783 (2006)

[5] D. Bigourd, L. Lago, A. Mussot, A. Kudlinski, J. F Gleyze, E. Hugonnot, "High-gain fiber, optical parametric chirped pulse amplification of femtosecond pulses at $1 \mu \mathrm{m}, "$ Opt. Lett. 35, 3480 (2010)

[6] D.G. Ouzounov, F.R. Ahmad, D. Muller, N. Venkataraman, M.T. Gallagher, M.G. Thomas, J. Silcox, K.W. Koch, A.L. Gaeta, "Generation of Megawatt Optical Solitons in Hollow-Core Photonic Band-Gap Fibers," Sciences, 301, 1702 (2003)

[7] C. Caucheteur, D. Bigourd, E. Hugonnot, P. Szriftgiser, A. Kudlinski, M. Gonzalez-Herraez, A.Mussot, "Experimental demonstration of optical parametric chirped pulse amplification in optical fiber," Opt. Lett. 35, 1786 (2010)

[8] Y. Zhou, Q. Li, K. K. Y. Cheung, S. Yang, P. C. Chui, K. Wong, "All-fiber based ultrashort pulse generation and chirped pulse amplification through parametric processes, IEEE Photon. Technol. Lett. 22, 1330 (2010) 
[9] V. Cristofori, Z. Lali-Dastjerdi, L. S. Rishj, M. Galili, C. Peucheretand, K. Rottwitt, "Dynamic characterization and amplification of sub-picosecond pulses in fiber optical parametric chirped pulse amplifiers," Opt. Express 21, 26044 (2013)

[10] K. Xu, H. Liu, Y. Dai, J. Wu, J. Lin, "Synthesis of broad band and Flat parametric gain by idler loss in optical fiber," Opt. Commun.285, 790 (2012)

[11] M. E. Marhic, F. S. Yang, M.-C. Ho, L. Kazovsky, "High nonlinearity fiber optical parametric amplifier with periodic dispersion compensation," J. Lightwave Technol. 17, 210 (1999)

[12] C.Fourcade-Dutin, Q. Bassery, D. Bigourd, A. Bendahmane, A. Kudlinski, M. Douay, A. Mussot, "12 THz flat gain fiber optical parametric amplifiers with dispersion varying fibers," Opt. Express 23, 10103 (2015)

[13] D. Bigourd, P. Beaure d'Augerès, J. Dubertrand, E. Hugonnot, A. Mussot, "Ultra-broad band fiber optical parametric amplifier pumped by chirped pulses," Opt. Lett. 13, 3782 (2014)

[14] M.E. Marhic, Y. Park, F.S. Yang, L.G. Kasovsky, "Broad band fiber-optical parametric amplifiers and wavelength converters with low-ripple Chebyshev gain spectra," Opt. Lett. 21, 1354 (1996)

[15] A. Vedadi, M.E. Marhic, E. Lantz, H. Maillotte, T. Sylvestre "Investigation of gain ripple in two-pump fiber optical parametric amplifiers," Opt. Lett. 33, 2203 (2008)

[16] M. Gao, C. Jiang, W. Hu, J. Wang, "Two-pump fiber optical parametric amplifiers with three-section fibers allocation," Opt. Laser Technol. 38, 186 (2006)

[17] A. Mussot, A. Kudlinski,P. Beaure dAugres, E. Hugonnot, "Amplification of ultra-short optical pulses in a two pump fiber optical parametric chirped pulse amplifier," Opt. Express 21, 12197 (2013)

[18] N. Ishii, C. Y. Teisset, S. Khler, E. E. Serebryannikov, T. Fuji, T. Metzger, F. Krausz, A. Baltuka, A. M. Zheltikov, "Widely tunable soliton frequency shifting of few-cycle laser pulses," Phys. Rev. E 74, 036617 (2006)
[19] A. M. Heidt, "Pulse preserving flat-top supercontinuum generation in all-normal dispersion photonic crystal fibers," J. Opt. Soc. Am. B 27550 (2010)

[20] M. Schultze, T. Binhammer, T. Binhammer, A. Steinmann, G. Palmer, M. Emons, U. Morgner, "Few-cycle OPCPA system at $143 \mathrm{kHz}$ with more than $1 \mu \mathrm{J}$ of pulse energy," Opt. Express 18, 2836 (2010)

[21] A.M. Al-Kadry, D. Strickland, "Generation of $400 \mu \mathrm{W}$ at $17,5 \mu \mathrm{m}$ using a two-color Yb fiber chirped pulse amplifier," Opt. Lett. 36, 1080 (2011)

[22] P. Morin, J. Dubertrand, P. Beaure d'Augères, G. Bouwmans, A. Kudinski, Y. Quiquempois, A. Mussot, E. Hugonnot, " $\mu \mathrm{J}$ range optical parametric chirped pulse amplification of short pulses at $1 \mu \mathrm{m}$," in Conference on Lasers and Electro-Optics, OSA Technical Digest (2016), paper STu1I.5

[23] M. Krebs, S. Hadrich, S. Demmler, J. Rothhardt, A. Zair, L. Chipperfield, J. Limpert, A. Tunnermann, "Towards isolated attosecond pulses at megahertz repetition rates," Nature Photonics 7555 (2013)

[24] O. Vanvincq, C. Fourcade-Dutin, A. Mussot, E. Hugonnot, D. Bigourd, "Ultra-broad band fiber optical parametric amplifier pumped by chirped pulses Part I : Analytical model," J. Opt. Soc. Am. B 321479 (2015)

[25] C. Fourcade-Dutin, O. Vanvincq, A. Mussot, E. Hugonnot, D. Bigourd, "Ultra-broad band fiber optical parametric amplifier pumped by chirped pulses Part II : Sub-30 fs pulse amplification at high gain," J. Opt. Soc. Am. B 321488 (2015)

[26] M. E. Marhic, Fiber Optical Parametric Amplifiers, Oscillators and Related Devices (Cambridge University, 2007).

[27] J. M. Chavez Boggio, J. D. Marconi, S. R. Bickham, H. L. Fragnito, "Spectrally flat and broad band doublepumped fiber optical parametric amplifiers," Opt. Express, 15, 5288 (2007) 